TRABAJO ORIGINAL

\title{
IMPACTO DE DIFERENTES TÉCNICAS BARIÁTRICAS SOBRE EL PERFIL LIPÍDICO EN OBESOS
}

\author{
IMPACT OF DIFFERENT BARIATRIC TECHNIQUES \\ ON THE LIPID PROFILE IN OBESES
}

\author{
Susana Gutt' ${ }^{1}$, María Soledad Saez², Axel Beskow³ , Ana Jesica Tamaroff ${ }^{4}$, María Mercedes Gutiérrez \\ María Victoria Lorenzón ${ }^{6}$, Patricia Sorroche
}

\section{RESUMEN}

Introducción: la obesidad es una enfermedad crónica de características epidémicas determinada, entre otras variables, por la interacción de factores genéticos y medioambientales. En pacientes obesos se presenta la dislipemia con un fenotipo aterogénico. La cirugía bariátrica (CB) constituye una alternativa terapéutica eficaz para la disminución del riesgo cardiovascular en tanto mejora el perfil lipídico.

Objetivos: comparar las diferencias en el perfil lipídico antes y después de someter a los pacientes del Hospital Italiano de Buenos Aires a dos técnicas distintas de cirugía bariátrica con un seguimiento al año de la intervención.

Materiales y métodos: en el presente estudio se incluyeron todos los pacientes sometidos a CB en el año $2015(n=138)$ en el Hospital Italiano de Buenos Aires.

Resultados: la reducción de los niveles de colesterol total, triglicéridos y LDL así como el aumento de HDL al año de la intervención fueron estadísticamente significativos y no se hallaron diferencias al comparar los deltas de cada variable del perfil lipídico entre dos técnicas quirúrgicas diferentes.

Conclusiones: la CB puede constituir un tratamiento efectivo en pacientes obesos con dislipidemia que no respondan a la terapia farmacológica dado que se observan cambios significativos en el perfil lipídico.

Palabras clave: cirugía bariátrica; triglicéridos; obesidad; HDL-colesterol.

Revista de la Sociedad Argentina de Diabetes 2018; Vol. 52 (71-74)

\section{ABSTRACT}

Introduction: obesity is a chronic disease of epidemic characteristics determined among other variables by the interaction of genetic and environmental factors. In patients with obesity an atherogenic phenotype is present.

Bariatric surgery (BS) is an effective therapeutic alternative for the reduction of cardiovascular risk while improving the lipid profile.

Objectives: to compare the differences in the lipid profile before and after submitting patients to two different bariatric surgery techniques with follow-up to the year of the intervention.

Materials and methods: in the present study were included all patients undergoing BS in $2015(n=138)$ at the Hospital Italiano de Buenos Aires.

Results: the reduction of total cholesterol, triglycerides and $L D L$ levels as well as the increase in HDL one year after the intervention were statistically significant and no differences were found when comparing the deltas of each variable of the lipid profile between two different surgical techniques.

Conclusions: BS can be an effective treatment in obese patients with dyslipidemia who do not respond to pharmacological therapy given that significant changes are observed in the lipid profile.

Key words: bariatric surgery; triglycerides; obesity; HDL-cholesterol. Revista de la Sociedad Argentina de Diabetes 2018; Vol. 52 (71-74)
Médica especialista en Nutrición, Jefa de la Sección Nutrición del Servicio de Clínica Médica, Hospital Italiano de Buenos Aires, Ciudad Autónoma de Buenos Aires, Argentina

2 Bioquímica, Laboratorio Central, Hospital Italiano de Buenos Aires, Ciudad Autónoma de Buenos Aires, Argentina

3 Médico Cirujano, Jefe de la Sección Cirugía Bariátrica, Servicio de Cirugía General, Hospital Italiano de Buenos Aires, Ciudad Autónoma de Buenos Aires, Argentina

4 Médica especialista en Nutrición, asociada a la Sección de Nutrición del Servicio de Clínica Médica, Hospital Italiano de Buenos Aires, Ciudad Autónoma de Buenos Aires, Argentina

5 Médica especialista en Nutrición, adscripta a la Sección de Nutrición del Servicio Clínica Médica, Hospital Italiano de Buenos Aires, Ciudad Autónoma de Buenos Aires, Argentina
6 Bioquímica, Laboratorio Central, Hospital Italiano de Buenos Aires, Ciudad Autónoma de Buenos Aires, Argentina

7 Bioquímica, Jefa de la Sección Proteínas y Lípidos del Laboratorio Central, Hospital Italiano de Buenos Aires, Ciudad Autónoma de Buenos Aires, Argentina

Contacto de la autora: Susana Gutt

E-mail: susana.gutt@gmail.com

Correspondencia: Tte. Gral. J. D. Perón 4190 (C1199ABB),

Ciudad Autónoma de Buenos Aires, Argentina

Fecha de trabajo recibido: 22/11/18

Fecha de trabajo aceptado: 31/01/19

Conflictos de interés: los autores declaran que no existe conflicto de interés 


\section{INTRODUCCIÓN}

La obesidad es una enfermedad crónica de características epidémicas determinada, entre otras variables, por la interacción de factores genéticos y medioambientales.

Se caracteriza por presentar enfermedades asociadas entre las que se distinguen las dependientes de alteraciones metabólicas como la dislipemia aterogénica, la diabetes mellitus tipo 2 (DM2), la hipertensión arterial y el hígado graso no alcohólico'.

En pacientes con obesidad y riesgo cardiovascular prevalece la dislipemia con fenotipo aterogénico. A pesar del uso de estatinas, indicadas ya sea por prevención primaria o secundaria, la tasa de eventos permanece elevada ${ }^{2}$, los fibratos tampoco reducen el riesgo aún en combinación con estatinas. Sí, en cambio, se han observado beneficios según recientes metaanálisis con ácidos grasos omega 3 en pacientes que reciben estatinas.

La cirugía bariátrica (CB) es una intervención sobre el tubo digestivo que logra el descenso del peso corporal y su mantenimiento a largo plazo. Los procedimientos practicados actualmente se clasifican según sus mecanismos de acción en restrictivos, gastroplastía en manga o manga gástrica (MG) y/o malabsortivos, by pass gástrico en $Y$ de Roux (BGYR). Estos procedimientos constituyen una alternativa terapéutica eficaz en pacientes con obesidad mórbida y con obesidad moderada asociada a comorbilidades metabólicas, especialmente DM2, y en la disminución del riesgo cardiovascular en tanto mejora el perfil lipídico ${ }^{3}$.

\section{OBJETIVOS}

Comparar las diferencias en los niveles de los componentes del perfil lipídico antes y después de dos técnicas distintas de cirugía quirúrgica.

\section{MATERIALES Y MÉTODOS}

El estudio se llevó a cabo en el Hospital Italiano de Buenos Aires por el equipo multidisciplinario de Cirugía Bariátrica responsable de la evaluación integral de los pacientes candidatos a CB desde el año 1999. Participaron médicos especialistas en Nutrición, psiquiatras y cirujanos especializados en cirugía bariátrica que realizan aproximadamente 140 cirugías por año.

Es un estudio de cohorte retrospectivo de 138 pacientes obesos a quienes se les efectuaron dos tipos de técnica quirúrgica de $\mathrm{CB}$.
Los pacientes fueron sometidos a una selección previa a la CB de acuerdo a los criterios de la American Society for Metabolic and Bariatric Surgery (ASMBS) ${ }^{4}$ y del Consenso Argentino de Nutrición en Cirugía Bariátrica ${ }^{5}$.

El objetivo principal de este estudio fue comparar el delta de los niveles plasmáticos de colesterol total (CT), HDL-col (HDL), LDL-col (LDL) y triglicéridos (TG) antes de la cirugía y al año de la misma entre pacientes a quienes se les realizó una u otra técnica quirúrgica.

Se incluyeron todos los pacientes sometidos a MG y a BGYR en el año 2015. El seguimiento fue desde la fecha de cirugía hasta los 12 \pm 3 meses postquirúrgicos. Las variables utilizadas se recolectaron a partir de la historia clínica electrónica. Se registraron los siguientes parámetros: sexo, edad, porcentaje del sobrepeso perdido (PSP), glucemia (G) y el perfil lipídico: CT, HDL, LDL y TG. Este último y la $G$ se determinaron por métodos enzimáticos en AU 5800 Beckman Coulter. EI LDL se calculó con la fórmula de Friedewald. El porcentaje de sobrepeso perdido (PSP) se calculó mediante la siguiente fórmula ${ }^{1}$ :

[(peso inicial-peso actual)/(peso inicial-peso ideal)] $\times 100$.

\section{Muestreo y cálculo muestral}

El muestreo fue consecutivo y dado que la muestra era fija no se realizó estimación del tamaño muestral.

\section{Análisis estadístico}

Las variables continuas se expresaron como media $\pm D S$ y las variables categóricas como número absoluto y porcentaje. Se calculó el delta de cada variable del perfil lipídico restando al valor de cada variable al año de CB el valor previo a la intervención quirúrgica. Se comparó la diferencia de las variables del perfil lipídico al año de una u otra técnica quirúrgica mediante un t-Test para muestras pareadas. Luego se calculó la media del delta de cada variable para cada grupo de pacientes según técnica quirúrgica y se compararon entre sí con t-Test para muestras no pareadas. Una $p<0,05$ fue considerada estadísticamente significativa. Para el análisis estadístico se utilizó PRISM 7.

El protocolo fue aprobado por el Comité de Ética Protocolos de Investigación (CEPI) del Hospital Italiano de Buenos Aires. 


\section{RESULTADOS}

Durante el año 2015 se sometieron a CB 138 (100\%) pacientes. A 91 de ellos (66\%) se les realizó BGYR y a 47 (34\%) MG. La totalidad de los pacientes logró un año de seguimiento y aportó los resultados del perfil lipídico en ambos tiempos.

En resumen, la población estudiada estuvo representada en un $69,5 \%$ por mujeres, con una edad media de 47 años.

La media de G previa a la CB fue de $112 \mathrm{mg} / \mathrm{dL}$ $(D S \pm 29,5)$ y de $101 \mathrm{mg} / \mathrm{dL}(\mathrm{DS} \pm 16,1)$, y al año de la intervención de $92 \mathrm{mg} / \mathrm{dL}(\mathrm{DS} \pm 11,2)$ y $86 \mathrm{mg} / \mathrm{dL}$ $(D S \pm 8,3)$ para los grupos sometidos a BGYR y MG respectivamente.

En la Tabla 1 se muestra la comparación entre las características de la población sometida a cada técnica quirúrgica; la única diferencia significativa fue la edad, y los pacientes sometidos a MG fueron significativamente más jóvenes.

La comparación de las variables del perfil lipídico antes y después de la cirugía así como su delta se detallan en las Tablas 2 y 3 . En ambos grupos, la reducción de los niveles de CT, TG y LDL colesterol como el aumento de HDL al año de la intervención fueron estadísticamente significativos.

Al comparar los deltas de cada variable del perfil lipídico entre una técnica quirúrgica y otra no se encontraron diferencias estadísticamente significativas (Tabla 4).

\begin{tabular}{|c|c|c|c|}
\hline \multirow[t]{2}{*}{ Variable } & BGYR & MG & \multirow[t]{2}{*}{$\mathbf{P}$} \\
\hline & $(\mathrm{N}=91)$ & $(\mathrm{N}=47)$ & \\
\hline Media edad-años $\pm \mathrm{DS}$ & $47(10,3)$ & $42(10,07)$ & 0,009 \\
\hline N mujeres (\%) & $59(64)$ & $35(75)$ & 0,308 \\
\hline Media IMC $\left(\mathrm{kg} / \mathrm{m}^{2}\right) \pm \mathrm{DS}$ & $43,1(6,15)$ & $43.45(4.4)$ & 0,742 \\
\hline $\begin{array}{l}\text { Colesterol total } \\
(\mathrm{mg} / \mathrm{dl}) \pm \mathrm{DS}\end{array}$ & $190(38,5)$ & $193(37,2)$ & 0,674 \\
\hline $\mathrm{LDL}(\mathrm{mg} / \mathrm{dl}) \pm \mathrm{DS}$ & $111(31,3)$ & $120(32,6)$ & 0,122 \\
\hline $\mathrm{HDL}(\mathrm{mg} / \mathrm{dl}) \pm \mathrm{DS}$ & $47(14)$ & $46.2(10.2)$ & 0,603 \\
\hline Triglicéridos $(\mathrm{mg} / \mathrm{dl}) \pm \mathrm{DS}$ & $172(145)$ & $142(74,8)$ & 0,219 \\
\hline
\end{tabular}

BGYR: by pass gástrico en Y de Roux; MG: manga gástrica.

Tabla 1: Comparación entre las características prequirúrgicas de los pacientes sometidos a una u otra técnica quirúrgica de CB en el Hospital Italiano de Buenos Aires, en el período comprendido de enero a diciembre de 2015 ( $n=138)$.

\begin{tabular}{|c|c|c|c|c|}
\hline Variable & $\begin{array}{c}\text { Valores pre- } \\
\text { quirúrgicos }\end{array}$ & $\begin{array}{c}\text { Valores } \\
\text { al año }\end{array}$ & Delta & P \\
\hline $\begin{array}{c}\text { Colesterol total } \\
(\mathrm{mg} / \mathrm{dl}) \pm \mathrm{DS}\end{array}$ & $193(37,2)$ & $181(35,6)$ & 9,6 & $<0,0197$ \\
\hline $\mathrm{LDL}(\mathrm{mg} / \mathrm{dl}) \pm \mathrm{DS}$ & $120(32,6)$ & $106(31,3)$ & 14,4 & $<0,0001$ \\
\hline $\mathrm{HDL}(\mathrm{mg} / \mathrm{dl}) \pm \mathrm{DS}$ & $46(10,2)$ & $54(12,2)$ & $-9,05$ & $<0,0001$ \\
\hline $\begin{array}{c}\text { Triglicéridos } \\
(\mathrm{mg} / \mathrm{dl}) \pm \mathrm{DS}\end{array}$ & $142(75)$ & $98(38)$ & 46,7 & $<0,0001$ \\
\hline
\end{tabular}

Tabla 2: Comparación de las variables del perfil lipídico antes y después de la cirugía en pacientes sometidos a manga gástrica $(n=47)$.

\begin{tabular}{|c|c|c|c|c|}
\hline Variable & $\begin{array}{c}\text { Valores pre- } \\
\text { quirúrgicos }\end{array}$ & $\begin{array}{c}\text { Valores } \\
\text { al año }\end{array}$ & Delta & $\mathbf{P}$ \\
\hline $\begin{array}{c}\text { Colesterol total } \\
(\mathrm{mg} / \mathrm{dl}) \pm \mathrm{DS}\end{array}$ & $191(38,4)$ & $165(25,9)$ & 21,8 & $<0,0001$ \\
\hline $\mathrm{LDL}(\mathrm{mg} / \mathrm{dl}) \pm \mathrm{DS}$ & $111(31,3)$ & $90(22,3)$ & 19,3 & $<0,0001$ \\
\hline $\mathrm{HDL}(\mathrm{mg} / \mathrm{dl}) \pm \mathrm{DS}$ & $47(14)$ & $56(14,2)$ & $-8,4$ & $<0,0001$ \\
\hline $\begin{array}{c}\text { Triglicéridos } \\
(\mathrm{mg} / \mathrm{dl}) \pm \mathrm{DS}\end{array}$ & $172(31,3)$ & $88(31,1)$ & 69,8 & $<0,0001$ \\
\hline
\end{tabular}

Tabla 3: Comparación de las variables del perfil lipídico antes y después de la cirugía en pacientes sometidos a by pass gástrico en $Y$ de Roux ( $n=91)$.

\begin{tabular}{|c|c|c|c|}
\hline Variable & Delta BGYR & Delta MG & \multirow{2}{*}{ P } \\
\cline { 2 - 3 } & $\mathbf{( N = 9 1 )}$ & $\mathbf{( N = 4 7 )}$ & \multirow{2}{*}{0,505} \\
\hline $\begin{array}{c}\text { Porcentaje del } \\
\text { sobrepeso perdido al } \\
\text { año } \pm D S(\%)\end{array}$ & $75,54(24,63)$ & $72,70(19,30)$ & \\
\hline $\begin{array}{c}\text { Colesterol total } \\
(\mathrm{mg} / \mathrm{dl}) \pm \mathrm{DS}\end{array}$ & $21,18(43,6)$ & $9,67(24,1)$ & 0,139 \\
\hline $\mathrm{LDL}(\mathrm{mg} / \mathrm{dl}) \pm \mathrm{DS}$ & $19,23(39,3)$ & $14,42(19,92)$ & 0,497 \\
\hline $\mathrm{HDL}(\mathrm{mg} / \mathrm{dl}) \pm \mathrm{DS}$ & $-8,45(17,8)$ & $-9,05(9,18)$ & 0,85 \\
\hline $\begin{array}{c}\text { Triglicéridos } \\
(\mathrm{mg} / \mathrm{dl}) \pm \mathrm{DS}\end{array}$ & $69,81(78,71)$ & $46,74(62,8)$ & 0,133 \\
\hline
\end{tabular}

BGYR: by pass gástrico en Y de Roux; MG: manga gástrica.

Tabla 4: Comparación de los deltas de cada variable del perfil lipídico entre ambas técnicas quirúrgicas ( $n=138)$.

\section{DISCUSIÓN}

Los lípidos complejos están formados por ácidos grasos, moléculas hidrófobas que se caracterizan por circular en sangre transportadas por lipoproteínas (LP). Las Lp se componen de una membrana hidrofílica que contiene fosfolípidos, 
apolipoproteínas y colesterol libre, y un núcleo hidrofóbico que posee TG y colesterol esterificado. Se diferencian según su densidad relativa, por su tamaño y su composición química ${ }^{6}$.

Las Lp de muy baja densidad (VLDL) son las que transportan mayor cantidad de TG. Las HDL tienen propiedades antiinflamatorias y antiaterogénicas dado que promueven el eflujo de colesterol de las paredes arteriales e inhiben la oxidación de las LDL. En la obesidad, la resistencia periférica a la acción de la insulina constituye una de las bases fisiopatológicas la cual favorece el aumento de peso, la disglucemia y la asociación con hipertrigliceridemia y concentraciones disminuidas de HDL.

Los efectos metabólicos de la CB, como el aumento de la sensibilidad a la insulina que sucede al descenso del peso corporal, impactan beneficiosamente sobre el perfil lipídico y en la cohorte estudiada no fueron afectados por el perfil glucémico.

Las guías de tratamiento recomiendan: cambio de estilo de vida, descenso de peso, dieta, actividad física y eventualmente farmacoterapia en pacientes con hipertrigliceridemia?.

Dado que la Food and Drugs Administration (FDA) en el año 2016 retiró la aprobación de niacina y algunos fibratos en combinación con el tratamiento con estatinas, en pacientes con obesidad y dislipidemia, la CB puede constituir un tratamiento, ya no sólo en relación al descenso de peso sino en la disminución del riesgo cardiovascular en tanto mejora el perfil lipídico.

En el presente estudio, al analizar los deltas de cada variable, no se obtuvieron diferencias significativas al comparar ambas técnicas; los lípidos plasmáticos mejoraron significativamente luego de la CB independientemente de la técnica quirúrgica implementada, si bien la literatura muestra resultados diversos en cuanto a las diferentes técnicas.

\section{CONCLUSIONES}

La CB puede constituir un tratamiento muy efectivo en pacientes obesos con dislipidemia dado que se observan cambios significativos en el perfil lipídico de estos pacientes independientemente de la técnica quirúrgica que se utilice.

\section{BIBLIOGRAFÍA}

1. Braguinsky J, y col. Obesidad: saberes y conflictos. Un tratado de obesidad. ACINDES 2007.

2. Bhatt $D L$, Steg G, Miller $M$, et al. Cardiovascular risk reduction with icosapent ethyl for hypertriglyceridemia. N Engl J Med 2019; 380:11-22.

3. Busetto L, Dicker D, Azran C, et al. Practical recommendations of the obesity management task force of the European Association for the Study of Obesity for the Post-Bariatric Surgery Medical Management. Obes Facts 2017;10(6):597-632.

4. Aills L, Blankenship J, Buffington C, et al. ASMBS Allied Health Nutritional Guidelines for the Surgical Weight Loss Patient. Surg Obes Relat Dis 2008 Sep-Oct; 4 (5 Suppl):S73-108.

5. Pampillón N, Reynoso C, Solimano M, Sánchez M, Ackerman M, y col. Actualización del Consenso Argentino de Nutrición en Cirugía Bariátrica. Revista Actualización en Nutrición marzo 2016; Vol. $17 \mathrm{~N}^{\circ} 1$ : 19-32.

6. Badellino KO, Wolfe ML, Reilly MP, Rader DJ. Endothelial lipase is increased in vivo by inflammation in humans. Circulation 2008; 117:678-685.

7. Grundy S, Stone NJ, Bailey AL, et al. 2018 AHA/ACC/AACVPR/ AAPA/ABC/ACPM/ADA/AGS/APhA/ASPC/NLA/PCNA Guideline on the Management of Blood Cholesterol: Executive Summary: A Report of the American College of Cardiology/American Heart Association Task Force on Clinical Practice Guidelines. J Am Coll Cardiol 2018 Nov 3; pii: S0735-1097(18)39033-8. Doi: 10.1016/j.jacc.2018.11.002. 